\title{
Morbidade hospitalar por doenças associadas à poluição do ar na cidade de Volta Redonda, Rio de Janeiro: casos e custo econômico
}

\author{
Hospital morbidity due to diseases associated with air pollution in the \\ city of Volta Redonda, Rio de Janeiro: cases and economic cost
}

\author{
Roberta Fernanda da Paz de Souza Paiva ${ }^{1}$
}

\begin{abstract}
Resumo
Este trabalho teve como objetivo estimar os casos de internação hospitalar por doenças respiratórias selecionadas associados aos níveis de poluição atmosférica na cidade de Volta Redonda (RJ), no período de 2005 a 2007 . Foi estimada uma função de regressão de Poisson a partir dos dados diários sobre Autorização de Internação Hospitalar (AlH) disponibilizados pelo Departamento de Informática do Sistema Único de Saúde (DATASUS) - variável dependente, níveis diários de poluentes encontrados na atmosfera coletados pelo Instituto Estadual do Ambiente (INEA) e variáveis de controle. Como principal resultado destaca-se que, das 5.235 internações pelo grupo de doenças avaliado, 6\% (317 casos) estão associadas aos níveis de poluentes encontrados na atmosfera. O custo econômico gerado pelas hospitalizações, captado por meio do Método dos Custos Evitados, foi de $\mathrm{R} \$ 170.711,83$. Concluiu-se que a existência de substâncias poluentes na atmosfera contribui para o acometimento da população por doenças respiratórias, levando a perdas de bem-estar e econômicas, além de elevar o dispêndio do Sistema Único de Saúde (SUS) com o tratamento e a recuperação das condições de saúde dos pacientes. Nesse contexto, faz-se imprescindível a adoção de políticas que reduzam/eliminem os níveis de poluentes na atmosfera e, em consequência, os prejuízos econômicos e desconfortos gerados.
\end{abstract}

Palavras-chave: internação hospitalar; poluição do ar; doenças respiratórias.

\begin{abstract}
This work aimed to estimate the cases of hospitalar internment for selected respiratory diseases associated to the levels of atmospheric pollution in the city of Volta Redonda (RJ), in the period from 2005 to 2007. A function of regression of Poisson was estimated from the daily data on the Hospitalar Internment Authorization (HIA) made available by DATASUS (dependent variable), daily levels of pollutants found in the atmosphere collected by the State Environment Institute (SEI) and control variables. As the main result it is emphasized that among the 5,235 internments, by the evaluated control group, 6\% (317 cases) are associated to the pollutants levels found in the atmosphere. The economic cost generated by these diseases, captured by the Methods of the Avoided Costs, was $R \$ 170,711.83$. It was concluded that the existence of pollutant substances in the atmosphere contributes respiratory diseases in the population taking to well-being and economic losses besides elevating the expenses of the Health Unique System (HUS) with the treatment and recuperation of the patients' health condition. In this context, the adoption of public politics that come to reduce/eliminate the levels of pollution in the atmosphere and, consequently, the generated economic damages and discomforts becomes indispensable.
\end{abstract}

Keywords: hospitalization; air pollution; respiratory tract diseases.

Trabalho realizado na Universidade Estadual de Campinas (UNICAMP) - Campinas (SP) e na Universidade Federal Fluminense (UFF) - Volta Redonda (RJ), Brasil. ${ }^{1}$ Graduada em Ciências Econômicas pela Universidade Federal de Juiz de Fora (UFJF); Doutora em Desenvolvimento Econômico pela UNICAMP; Professora Adjunta da UFF - Volta Redonda (RJ), Brasil.

Endereço para correspondência: Roberta Fernanda da Paz de Souza Paiva - Universidade Federal Fluminense - Avenida dos Trabalhadores, 420 - Vila Santa Cecília - CEP: 27255-125 - Volta Redonda (RJ), Brasil - E-mail: robertapaz2003@yahoo.com.br

Fonte de financiamento: nenhuma.

Conflito de interesses: nada a declarar. 


\section{INTRODUÇÃO}

$\mathrm{O}$ atendimento das demandas geradas pelo crescimento econômico e a ampliação das necessidades de consumo da população dão uma nova dinâmica ao contexto de exploração do meio ambiente. Com o passar do tempo, níveis de renda, hábitos e culturas vão se modificando, o que leva à alteração $\mathrm{e}$ à elevação dos padrões de consumo. Permanentes transformações levam à necessidade de adaptações - econômica, social e política - e geram alterações no ambiente, sendo que essas adaptações também provocam outras mudanças ambientais (coevolutionary process).

Nesse contexto, um dos principais impactos observados está associado à perda das condições de saúde e qualidade de vida da população, devido a sua exposição a níveis de substâncias poluentes lançadas nos recursos naturais. A partir do momento em que são estabelecidas relações entre a poluição ambiental e casos de doenças relacionadas, é necessário direcionar ações públicas e privadas, no sentido de controlar os níveis de poluição e mitigar seus efeitos nocivos sobre a população.

Um dos problemas ambientais que mais preocupam a sociedade é a poluição atmosférica, que acarreta em impactos sobre a saúde humana, perdas econômicas, entre outros. No Brasil, de setembro de 2012 a agosto de 2013, tem-se que cerca de $10 \%$ dos gastos do Sistema Único de Saúde (SUS) com internações hospitalares foram destinados ao pagamento de internações para o tratamento de doenças respiratórias. Esse gasto foi inferior apenas àqueles destinados ao pagamento de internações para o tratamento de doenças do aparelho circulatório $(19,8 \%)$ e as associadas à gravidez, ao parto e ao puerpério $(10,6 \%)^{1}$.

Diversos estudos já foram desenvolvidos com vistas a avaliar a associação entre a poluição do ar e casos de morbidade e/ou mortalidade humana em diversas localidades ${ }^{2-9}$, sendo seus resultados importantes para determinar principais doenças e poluentes relacionados, a existência de grupos e fatores de risco, etc., facilitando o direcionamento das políticas públicas voltadas à resolução do problema ambiental em questão.

Este estudo teve como objetivo estimar os casos de internação hospitalar por doenças respiratórias selecionadas associados aos níveis de poluição atmosférica na cidade de Volta Redonda (RJ), no período de 2005 a 2007.

Na cidade está instalada a Companhia Siderúrgica Nacional (CSN), maior siderúrgica da América Latina, o que faz com que o setor industrial seja o mais representativo para a economia local, apesar do desenvolvimento do comércio e do setor de serviços.

Segundo estudo realizado pelo Instituto Brasileiro de Geografia e Estatística (IBGE), Volta Redonda é a segunda cidade do Rio de Janeiro com o maior potencial poluidor, ficando apenas atrás da capital do Estado ${ }^{10}$.
Esse resultado se deve às atividades industriais concentradas na cidade, com destaque para as atividades de metalurgia e de minerais não metálicos. Além dos poluentes emitidos pelas atividades industriais desenvolvidas na cidade e região, são emitidas grandes quantidades de poluentes devido ao intenso tráfego de veículos.

Segundo dados do Departamento de Informática do Sistema Único de Saúde (DATASUS) ${ }^{1}$, na cidade de Volta Redonda, no período de agosto de 2012 a setembro de $2013,9 \%$ do total gasto com o pagamento de Autorização de Internação Hospitalar (AIH) foram destinados àquelas para o tratamento de doenças do sistema respiratório, valor inferior apenas aos gastos para o tratamento de doenças do aparelho circulatório (27\%).

A análise se justifica pela falta de informações sobre a questão avaliada e pela necessidade de conhecimento para o embasamento de políticas sociais, ambientais e econômicas mais eficazes no que se refere à poluição do ar e seus impactos.

\section{METODOLOGIA}

Para que o número de casos associados à poluição do ar e os gastos incorridos com o tratamento das mesmas sejam calculados, deve ser estimada uma função dose-resposta. A estimação de tal função constitui-se num estudo ecológico de séries temporais que se caracteriza por envolver um número de eventos ocorridos de uma determinada variável (por exemplo, doenças respiratórias), num dado período de tempo, em uma região geográfica definida. Seu objetivo principal é determinar uma possível associação entre as variações na intensidade da exposição e na frequência das doenças ${ }^{11}$.

No caso do presente estudo, foi avaliada a associação entre o número de casos de doenças respiratórias (variável dependente) com as concentrações dos poluentes selecionados emitidos na atmosfera e as condições de temperatura (variáveis independentes), por meio da estimação do modelo de regressão de Poisson, modelo aplicável nos casos em que o regressando é do tipo contável ${ }^{12}$. O número diário de internações é um evento de contagem que, em geral, apresenta distribuição de Poisson, o que justifica a escolha do referido modelo para este estudo.

A equação representativa para tal modelo é:

$\ln \lambda_{t}=\alpha+\sum_{i=1}^{m} \beta_{i} X_{i t}$

onde: $\ln \lambda_{t}$ é o logaritmo natural da variável dependente (no caso do presente estudo, o número diário de internações), $X_{i t}$ corresponde às variáveis independentes, $\alpha$ e $\beta_{i}$ são parâmetros a serem estimados ${ }^{13}$.

O modelo de regressão de Poisson é um caso particular dos Modelos Lineares Generalizados (MLG), método selecionado 
para a estimação da função dose-resposta no presente trabalho $^{14}$. A estimação foi feita a partir do pacote estatístico Statistical Package for the Social Sciences (SPSS), versão 18.0.

Esses modelos são uma extensão dos modelos de regressão linear e se diferenciam dos mesmos por permitir que a variável resposta do modelo venha de um universo que siga uma lei de distribuição da família exponencial.

A variável representativa $(\mathrm{AIH})$ dos casos da doença será o número de internações hospitalares para as doenças do Capítulo $\mathrm{X}$ Doenças do aparelho respiratório - da Classificação Internacional de Doenças (CID 10): faringite aguda e amigdalite aguda, laringite e traqueíte agudas, outras infecções agudas das vias aéreas superiores, influenza (gripe), pneumonia, bronquite aguda e bronquiolite aguda, sinusite crônica, outras doenças do nariz e dos seios paranasais, doenças crônicas das amígdalas e das adenoides, outras doenças do trato respiratório superior, bronquite, enfisema e outras doenças pulmonares obstrutivas crônicas, asma, bronquiectasia, pneumoconiose, outras doenças do aparelho respiratório. $\mathrm{O}$ uso dessa variável se justifica pelo fato de não existir uma base de dados completa para todos os gastos realizados pelos indivíduos acometidos pelas doenças respiratórias.

A inclusão das doenças crônicas no estudo, que considera apenas lags curtos em sua aplicação, se deve à existência de pesquisas que indicam relação entre a exposição aguda de pacientes portadores de doenças pulmonares crônicas à poluição e o aumento das internações hospitalares.

Os dados utilizados referem-se aos casos de morbidade registrados pelo SUS por meio do DATASUS. Tais observações foram extraídas a partir dos registros de pagamentos efetuados pelo SUS. Dentre outras informações, os registros contêm a data de internação de cada paciente e a doença diagnosticada que justifica a internação.

Foram coletados dados referentes ao número diário de internações por doenças do aparelho respiratório na cidade de Volta Redonda (apenas para a rede pública), no período de $1^{\circ}$ de janeiro de 2005 até 31 de dezembro de 2007. Esses dados indicam o comportamento sazonal das internações, em que os picos ocorrem nos meses do outono e do inverno, com máximas de atendimentos nos meses de maio, junho e julho, respectivamente.

Para a estimação da função dose-resposta foram consideradas como variáveis independentes as concentrações diárias médias dos seguintes poluentes: partículas totais em suspensão PTS $\left(\mu \mathrm{g} / \mathrm{m}^{3}\right)$, monóxido de carbono - CO ( $\left.\mathrm{ppm}\right)$, partículas suspensas menores que $10 \mu \mathrm{g} / \mathrm{m}^{3}$ - PM-10 $\left(\mu \mathrm{g} / \mathrm{m}^{3}\right)$ e ozônio $\mathrm{O}_{3}\left(\mu \mathrm{g} / \mathrm{m}^{3}\right)$. Os dados diários referentes às concentrações de poluentes foram disponibilizados pelo Instituto Estadual do Ambiente (INEA). Os dados analisados indicam que a concentração diária dos poluentes avaliados não ultrapassou o padrão diário determinado pela legislação.

Foram incluídas como variáveis de controle: mês, a fim de controlar os efeitos da sazonalidade e a tendência; temperatura mínima $\left({ }^{\circ} \mathrm{C}\right)$ e variáveis representativas dos referidos dias e dos lags (defasagem) dos poluentes, buscando comprovar a associação entre as internações por doenças respiratórias (ou as manifestações dos efeitos da poluição sobre a saúde); e a concentração de poluentes do referido dia e também dos dias anteriores. Neste estudo foi analisada uma estrutura de defasagem variando de 1, 2, 3 e 7 dias.

O cálculo do risco relativo (RR), que, neste caso, se refere ao risco de elevação de internação hospitalar por doenças respiratórias, foi feito a partir $\mathrm{de}^{3,15}$ :

$R R=e^{\beta x}$

onde: $\beta$ é o parâmetro estimado pela regressão $\mathrm{e} X$ a variação de um interquartil (VIQ) nas concentrações de poluentes.

O intervalo de confiança do RR é dado pela seguinte equação ${ }^{15,3}$ :

IC95\% $(\mathrm{RR})=\mathrm{e}^{\left[\left(\beta^{*} \chi\right) \pm\left(1,96^{*}\left(\mathrm{ep}(\beta)^{*}\right)\right)\right]}$

onde: ep é o erro padrão de $\beta$.

Outra estimativa calculada foi a do acréscimo no número de internações dadas as variações interquartil no poluente CO (lag2). Tal acréscimo pode ser estimado por meio da seguinte equação ${ }^{3,15}$ :

$\mathrm{A}(\%)=\left(\mathrm{e}^{\beta \mathrm{\gamma}-1}\right) \times 100$

onde: X é a VIQ do poluente que se quer estimar e $\beta$ é o parâmetro estimado pela regressão de Poisson.

A estimativa do número de casos atribuíveis ao fator de risco (neste caso, a exposição ao poluente atmosférico) pode ser calculada a partir de $\mathrm{e}^{15,16}$ :

Estimativa do número de casos atribuíveis ao fator de risco $(\mathrm{NA})=$ risco atribuível populacional $\mathrm{x}$ número total de casos (5)

Sendo o risco atribuível populacional (RAP):

$\mathrm{RAP}=\frac{\mathrm{RR}-1}{\mathrm{RR}}$

Tem-se que:

$\mathrm{NA}=(\underline{\mathrm{RR}-1)}) \mathrm{xN}$

$\mathrm{RR}$

onde: NA é o número de casos atribuídos à concentração do poluente analisado, RR relaciona-se às internações devido à poluição e $\mathrm{N}$ é o número de internações por doenças respiratórias no período. 


\section{RESULTADOS}

A partir da determinação das variáveis que irão compor o modelo estimado, passou-se à análise das mesmas. O primeiro passo foi o cálculo da correlação de Pearson entre a variável dependente (AIH) e as variáveis ambientais (poluentes e temperatura).

Posteriormente, foram estimadas regressões de Poisson univariadas (sem a inclusão das variáveis de controle) para cada uma das variáveis propostas, com o objetivo de avaliar a possível associação entre as mesmas e a variável dependente AIH (Tabela 1).

Os resultados apresentados indicam que as variáveis citadas têm associação com a variável dependente. As variáveis CO lag7 e PTS lag7, apesar de não apresentarem coeficientes de correlação significativos, apresentaram coeficientes significativos nas estimações das regressões univariadas e, por isso, foram incluídas no modelo multivariado.

A variável $\mathrm{O}_{3}$ apresentou sinal negativo e não significância estatística para observações do dia da internação e para os dados defasados não sendo, então, inserida como explicativa das internações associadas à poluição atmosférica.

Foi realizado ainda o teste de Kolmogorov-Smirnov, para testar a não normalidade das variáveis.

Após a seleção das variáveis que seriam inseridas no modelo, foi estimada a regressão de Poisson multivariada, a partir do modelo linear generalizado (Tabela 2).

Segundo os resultados, tem-se que apenas as variáveis PM10 com a defasagem de um dia, a variável CO com a defasagem de dois dias e a variável PTS com defasagem para sete dias se mostraram significativas, indicando que, dentre os poluentes estudados, o monóxido de carbono, o material particulado e as partículas totais em suspensão foram os que mais afetaram a contagem de internações por doenças respiratórias no período estudado. Infere-se, ainda, que as concentrações mais significativas são aquelas defasadas, indicando que os impactos da exposição humana aos poluentes não são percebidos imediatamente, mas após um lapso de tempo.

Esse resultado não indica que os outros poluentes não contribuam de alguma forma para a morbidade, mas apenas que tais compostos não se mostraram significativos estatisticamente.

Após a identificação das variáveis significativas, foi estimada uma regressão restrita contendo apenas as variáveis de controle e as representativas da concentração de CO (lag2), PM10 (lag1) e PTS (lag7). Os coeficientes estimados são mostrados na Tabela 3.

A estimação da equação restrita confirma a associação entre a morbidade e a concentração defasada do poluente CO, mas não apresenta significância da relação entre as internações e o PM10 e PTS.
Seguindo o mesmo princípio, foi estimada uma regressão contendo as variáveis de controle e a variável significativa CO (lag2) (Tabela 4).

A variável representativa da temperatura mínima (média diária) mostrou-se significativa em todas as estimações, comprovando sua associação com as doenças respiratórias.

A partir dos resultados encontrados, puderam ser quantificados os impactos do poluente (monóxido de carbono) sobre a saúde humana, por meio da análise dos níveis de internação por doenças respiratórias.

Tabela 1. Correlação de Pearson e resultado da regressão univariada para as variáveis selecionadas

\begin{tabular}{|c|c|c|c|c|}
\hline \multirow{2}{*}{\multicolumn{2}{|c|}{ Variável independente }} & \multirow{3}{*}{$\begin{array}{c}\mathbf{r}(\mathrm{p}) \\
0,079^{*}\end{array}$} & \multicolumn{2}{|c|}{ Coeficientes de regressão } \\
\hline & & & \multirow{2}{*}{$\begin{array}{c}\alpha \\
1,458^{*}\end{array}$} & \multirow{2}{*}{$\begin{array}{c}\beta_{1} \\
0,004^{*}\end{array}$} \\
\hline PM10 & Dia & & & \\
\hline PM10 & $\operatorname{lag} 1$ & $0,092^{*}$ & $1,442^{*}$ & $0,004^{*}$ \\
\hline PM10 & $\operatorname{lag} 2$ & $0,097^{*}$ & $1,440^{*}$ & $0,004^{*}$ \\
\hline PM10 & $\operatorname{lag} 3$ & $0,096^{\star}$ & $1,427^{\star}$ & $0,005^{\star}$ \\
\hline PM10 & $\operatorname{lag} 7$ & $0,038^{\star}$ & $1,487^{*}$ & $0,003^{*}$ \\
\hline $\mathrm{CO}$ & Dia & $0,093^{*}$ & $1,471^{*}$ & $0,249^{*}$ \\
\hline $\mathrm{CO}$ & $\operatorname{lag} 1$ & $0,102^{*}$ & $1,459^{*}$ & $0,273^{*}$ \\
\hline $\mathrm{CO}$ & $\operatorname{lag} 2$ & $0,130^{*}$ & $1,432^{*}$ & $0,344^{*}$ \\
\hline $\mathrm{CO}$ & $\operatorname{lag} 3$ & $0,118^{*}$ & $1,437^{\star}$ & $0,340^{*}$ \\
\hline $\mathrm{CO}$ & $\operatorname{lag} 7$ & $0,047^{\mathrm{NS}}$ & $1,507^{\star}$ & $0,167^{\star}$ \\
\hline PTS & Dia & $0,102^{\star}$ & $1,433^{*}$ & $0,002^{*}$ \\
\hline PTS & $\operatorname{lag} 1$ & $0,092^{*}$ & $1,445^{*}$ & $0,002^{*}$ \\
\hline PTS & $\operatorname{lag} 2$ & $0,103^{\star}$ & $1,427^{\star}$ & $0,002^{*}$ \\
\hline PTS & $\operatorname{lag} 3$ & $0,127^{\star}$ & $1,388^{*}$ & $0,003^{*}$ \\
\hline PTS & $\operatorname{lag} 7$ & $0,075^{\mathrm{NS}}$ & $1,468^{*}$ & $0,002^{*}$ \\
\hline
\end{tabular}

*Significativo a $1 \%$.

NS: não significativo.

Tabela 2. Coeficientes de regressão e erro padrão para internações por doenças respiratórias no município de Volta Redonda, no período de janeiro de 2005 a dezembro de 2007, controlados para dia da semana, dias transcorridos, mês e temperatura mínima

\begin{tabular}{|c|c|c|c|}
\hline \multicolumn{2}{|c|}{ Variável independente } & \multirow{2}{*}{$\begin{array}{c}\text { Coeficientes } \\
\text { de regressão }(\beta) \\
2,266^{*}\end{array}$} & \multirow{2}{*}{$\begin{array}{c}\text { Erro padrão } \\
0,1259\end{array}$} \\
\hline$\alpha$ & - & & \\
\hline PM10 & Dia & $-0,002^{\mathrm{NS}}$ & 0,0023 \\
\hline PM10 & lag1 & $0,004^{\star *}$ & 0,0025 \\
\hline PM10 & $\operatorname{lag} 2$ & $-0,002^{\mathrm{NS}}$ & 0,0025 \\
\hline PM10 & $\operatorname{lag} 3$ & $0,001^{\mathrm{NS}}$ & 0,0023 \\
\hline PM10 & $\operatorname{lag} 7$ & $-0,001^{\mathrm{NS}}$ & 0,0021 \\
\hline $\mathrm{CO}$ & Dia & $0,008^{\mathrm{NS}}$ & 0,1154 \\
\hline $\mathrm{CO}$ & $\operatorname{lag} 1$ & $0,037^{\mathrm{NS}}$ & 0,1239 \\
\hline $\mathrm{CO}$ & lag2 & $0,233^{* *}$ & 0,1240 \\
\hline $\mathrm{CO}$ & $\operatorname{lag} 3$ & $-0,060^{\mathrm{NS}}$ & 0,1139 \\
\hline $\mathrm{CO}$ & $\operatorname{lag} 7$ & $-0,139^{\mathrm{NS}}$ & 0,1032 \\
\hline PTS & Dia & $0,001^{\mathrm{NS}}$ & 0,0012 \\
\hline PTS & $\operatorname{lag} 1$ & $0,004^{\mathrm{NS}}$ & 0,0013 \\
\hline PTS & $\operatorname{lag} 2$ & $0,001^{\mathrm{NS}}$ & 0,0012 \\
\hline PTS & $\operatorname{lag} 3$ & $0,001^{\mathrm{NS}}$ & 0,0012 \\
\hline PTS & lag7 & $0,002^{\star *}$ & 0,0011 \\
\hline
\end{tabular}

*Significativo a $1 \%$; ** significativo a $10 \%$. NS: não significativo. 
O RR e o intervalo de confiança de internação hospitalar por doenças respiratórias relacionadas à variação interquartil do monóxido de carbono encontrados foram, respectivamente, de 1,06438 e $(1,0316 ; 1,0980)$. O resultado indica que as variações interquartil ( $0,221 \mathrm{ppm} \mathrm{CO})$ aumentam o $\mathrm{RR}$ das admissões hospitalares por doenças respiratórias.

Para esse dado tem-se que um aumento de 0,221 ppm de CO está associado a um acréscimo de 6,35\% nas internações por doenças respiratórias.

A análise desses resultados confirma a associação entre as internações e a exposição à concentração de monóxido de carbono, já que a elevação de sua concentração aumenta o número de internações por doenças respiratórias.

A partir dos resultados obtidos e visando cumprir o objetivo proposto (calcular os custos associados à poluição do ar), foi ainda calculado, para o período estudado, o número de internações associadas à poluição do ar em Volta Redonda.

Com base na análise dos dados mensais de internação para o período deste estudo (janeiro de 2005 a dezembro de 2007), tem-se que 317 das 5.235 internações (6\% das doenças respiratórias no período) estão associadas aos níveis de $\mathrm{CO}$ encontrados na atmosfera.

Por meio do cálculo do número de internações associadas à poluição do ar pode-se estimar o custo dessa poluição, montante que seria encontrado por meio da multiplicação do valor médio da AIH pelo número de AIH (Tabela 5).

\section{DISCUSSÃO E CONSIDERAÇÕES FINAIS}

A análise dos resultados indica que a poluição atmosférica na cidade de Volta Redonda, no período estudado, está relacionada a internações por doenças respiratórias, principalmente pela exposição dos indivíduos ao monóxido de carbono.

Outro fator importante se refere ao fato de que essa associação foi comprovada mesmo quando níveis de poluentes não ultrapassaram o limite estabelecido pela legislação, indicando que os mesmos são nocivos ainda que em quantidades permitidas.

Além disso, a existência de correlação positiva entre as variáveis independentes e os resultados encontrados em outros estudos indica que os demais poluentes também estão associados às doenças respiratórias, apesar de não se mostrarem significativos nas análises estatísticas para o período em questão.

Em outros estudos essas associações foram comprovadas. Freitas et al. ${ }^{15}$ encontraram significância entre a associação de PM10 e as internações por doenças respiratórias na infância e a morte de idosos para a cidade de São Paulo. Schwartz et al. ${ }^{9}$ encontraram associação entre admissões hospitalares e a exposição a níveis de $\mathrm{O}_{3}$, $\mathrm{PM} 10$ e $\mathrm{SO}_{2}$. Gouvea et al. ${ }^{17}$
Tabela 3. Coeficientes de regressão e erro padrão do modelo restrito para internações por doenças respiratórias no município de Volta Redonda, no período de janeiro de 2005 a dezembro de 2007, controlados para dia da semana, dias transcorridos, mês e temperatura mínima

\begin{tabular}{llcc} 
Variável independente & $\begin{array}{c}\text { Coeficientes } \\
\text { de regressão }(\beta)\end{array}$ & Erro padrão \\
$\beta_{0}$ & - & $2,234^{*}$ & 0,1083 \\
PM10 & $\operatorname{lag} 1$ & $0,002^{\mathrm{NS}}$ & 0,0014 \\
PTS & $\operatorname{lag} 7$ & $0,001^{\mathrm{NS}}$ & 0,0007 \\
CO & $\operatorname{lag} 2$ & $0,227^{*}$ & 0,0819 \\
\hline
\end{tabular}

*Significativo a $1 \%$.

Tabela 4. Coeficientes de regressão e erro padrão do modelo restrito (CO lag2) para internações por doenças respiratórias no município de Volta Redonda, no período de janeiro de 2005 a dezembro de 2007, controlados para dia da semana, dias transcorridos, mês e temperatura mínima

\begin{tabular}{lccc}
\multicolumn{2}{l}{ Variável independente } & $\begin{array}{c}\text { Coeficientes } \\
\text { de regressão }(\beta)\end{array}$ & Erro padrão \\
$\beta_{0}$ & - & $2,332^{*}$ & 0,0973 \\
$\mathrm{CO}$ & $\operatorname{lag} 2$ & $0,282^{*}$ & 0,0720 \\
\hline
\end{tabular}

${ }^{*}$ Significativo a $1 \%$.

Tabela 5. Estimativa do custo associado à poluição do ar na cidade de Volta Redonda, Rio de Janeiro, nos anos de 2005, 2006 e 2007

\begin{tabular}{lccc} 
Ano & Total & Valor médio AIH* & Valor total \\
2005 & 105 & $\mathrm{R} \$ 487,71$ & $\mathrm{R} \$ 51.209,55$ \\
2006 & 106 & $\mathrm{R} \$ 507,56$ & $\mathrm{R} \$ 53.801,36$ \\
2007 & 106 & $\mathrm{R} \$ 619,82$ & $\mathrm{R} \$ 65.700,92$ \\
Total & 317 & & $\mathrm{R} \$ 170.711,83$ \\
\hline
\end{tabular}

*Valor médio das internações por doenças respiratórias, segundo dados do Sistema Único de Saúde.

concluíram em seu estudo sobre o impacto de $\mathrm{PM} 10, \mathrm{CO}, \mathrm{SO}_{2}$ e $\mathrm{NO}_{2}$ sobre doenças do aparelho circulatório e respiratório em crianças e idosos.

Além da associação às doenças, foram avaliados os gastos monetários incorridos pelo SUS para o tratamento dos doentes. Apesar de considerar apenas parte do custo gerado pelos desconfortos causados pela poluição do ar nos habitantes da cidade, a metodologia aplicada permite quantificar em termos monetários quanto recurso poderia ser direcionado a políticas públicas em prol da sociedade caso os níveis de poluição do ar não contribuíssem para a ocorrência das doenças.

Outros gastos (como o da rede privada de saúde, os gastos com medicamentos pelos pacientes, os dias de trabalho perdidos, entre outros) deveriam ser também quantificados, para que se desenhasse um cenário mais fidedigno e capaz de embasar políticas mais eficientes.

Deve ser enfatizado que, antes de se trabalhar com vistas a reduzir os gastos monetários, devem ser priorizadas as ações que restaurem os níveis satisfatórios de qualidade de vida e bem-estar da população. 


\section{REFERÊNCIAS}

1. Departamento de Informática do Sistema Único de Saúde. Informações de Saúde. Indicadores de Saúde [Internet]. Morbidade Hospitalar por residência da CID-10. [cited 2013 out 10] Available from: http://datasus.gov.br

2. Braga ALF, Conceição GMS, Pereira LAA, Kihi H, Pereira JCR, Andrade MF, et al. Air pollution and pediatric respiratory hospital admissions in São Paulo, Brazil. J Environ Med. 1999;2(1):95-102.

3. Cançado JED. A poluição atmosférica e sua relação com a saúde humana na região canavieira de Piracicaba SP [tese]. São Paulo (SP): Universidade de São Paulo; 2003.

4. Coelho MSZS. Uma análise estatística com vistas a previsibilidade de internações por doenças respiratórias em funções de condições meteorotrópicas na cidade de São Paulo [tese]. São Paulo (SP): Universidade de São Paulo; 2007.

5. Conceição GMS, Saldiva PHN, Singer JM. Modelos MLG e MAG para análise da associação entre poluição atmosférica e marcadores de morbimortalidade: uma introdução baseada em dados da cidade de São Paulo. Rev Bras Epidemiol. 2001;4(3):206-19.

6. Saldiva PH, Pope CA 3rd, Schwartz J, Dockery DW, Lichtenfels AJ, Salge JM, et al. Air pollution and daily mortality in elderly people: a time-series study in São Paulo, Brasil. Arch Environ Health. 1995;50(2):159-63.

7. Schwartz J. Air pollution and daily mortality in Birmingham, Alabama. Am J Epidemiol. 1993;137(10):1136-47.

8. Schwartz J. Air pollution and daily mortality: a review and meta analysis. Environ Res. 1994;64(1):36-52.

9. Schwartz J, Dockery DW, Neas LM. Is daily mortality associated specifically with fine particles? J Air Waste Manag Assoc. 1996;46(10):927-39.
10. Sor JL, Clevelário Júnior J, Guimarães LT, Moreno RAM. Relatório piloto com aplicação da metodologia IPPS ao estado do Rio de Janeiro: uma estimativa do potencial de poluição industrial do ar. Rio de Janeiro: Instituto Brasileiro de Geografia e Estatística; 2008.

11. Morgenstern $\mathrm{H}$. Uses of ecological analysis in epidemiologic research. Am J Public Health. 1982;72(12):1336-44.

12. Gujarati DN. Econometria Básica. Rio de Janeiro: Elsevier; 2006.

13. Kleinbaum DG, Kupper LL, Müller KE. Applied regression analysis and other multivariate methods. Belmont: Duxbury Press; 1988.

14. Simas HS. Aspectos metodológicos em análises de séries temporais epidemiológicas do efeito da poluição atmosférica na saúde pública: uma revisão bibliográfica e um estudo comparativo via simulação [dissertação]. Rio de Janeiro (RJ): Universidade do Estado do Rio de Janeiro; 2003.

15. Freitas CU, Pereira LAA, Saldiva PHN. Vigilância dos efeitos na saúde decorrentes da poluição atmosférica: estudo de factibilidade; 2002 [Internet]. [cited 2009 Dec 10]. Available from: www.cve.saude.sp.gov. $\mathrm{br} / \mathrm{htm} /$ doma_vig.htm

16. Camey SA, Agranonik M, Radaelli J, Hirakata VN. Fração Atribuível Populacional. Rev HCPA \& Fac Med Univ Fed Rio Gd do Sul. 2010; 30(1):77-85.

17. Gouvea N, Mendonça GAS, Ponce-de-Leon A, Correia JEM, Junger WL, Freitas CU, et al. Poluição do ar e efeitos na saúde nas populações de duas grandes metrópoles brasileiras. Epidemiol Serv Saúde. 2003;12(1):29-40.

Recebido em: 05/11/2013 Aprovado em: 17/05/2014 